\title{
ECONOMÍA CIRCULAR: DESAFÍOS PARA UNA VISIÓN ESTRATÉGICA DE LAS
}

\author{
EMPRESAS EXPORTADORAS
}

\section{ARTICLE CIRCULAR ECONOMY: CHALLENGES FOR A STRATEGIC VISION OF EXPORTING COMPANIES}

\author{
Nathaly Díaz ${ }^{1}$, Rita Menoscal ${ }^{2}$, Mayiya González ${ }^{3}$
}

Palabras clave:
Economía
circular,
Empresas
exportadoras,
Ventaja
competitiva,
Comercio
internacional,
Estrategia de
economía circular
de la Unión
Europea.

\section{Resumen}

La preocupación por la sostenibilidad del medio ambiente se hace cada vez fuerte en el ámbito de los negocios internacionales y las decisiones gubernamentales. La "Estrategia de Economía Circular" es un ejemplo de iniciativa legislativa por parte de la Unión Europea, que refleja pautas sobre la regulación del consumo de los recursos y las futuras relaciones con sus proveedores. Esta investigación tiene como propósito analizar la economía circular como estrategia competitiva para las empresas exportadoras, a partir de la revisión de 15 artículos científicos basados en investigaciones a empresas que han implementado la economía circular. El desarrollo de esta investigación se fundamentó bajo un enfoque cualitativo con la aplicación de los métodos analítico-sintético e histórico-lógico. Los resultados indican que las empresas están haciendo cambios en sus procesos productivos para contribuir a la disminución del uso de recursos y mejorar la gestión de residuos. Se concluye que las empresas, con ritmos diferentes, en función de sus fortalezas y capacidades, están consiguiendo desarrollar propuestas de valor para apuntalar la sostenibilidad de los recursos, logrando diferenciarse de sus competidores.

Códigos JEL: F18, L21, M16

\footnotetext{
${ }^{1}$ Universidad Técnica de Machala, Facultad de Ciencias Empresariales (Campus Central), Av. Panamericana Km. 5 $1 / 2$ Vía a Pasaje, Machala, Ecuador

E-mail: ngdiaz_est@utmachala.edu.ec

${ }^{2}$ Universidad Técnica de Machala, Facultad de Ciencias Empresariales (Campus Central), Av. Panamericana Km. 5 1/2

Vía a Pasaje, Machala, Ecuador

E-mail: rmenoscal_est@utmachala.edu.ec

${ }^{3}$ Universidad Técnica de Machala, Facultad de Ciencias Empresariales (Campus Central), Av. Panamericana Km. 5 1/2

Vía a Pasaje, Machala, Ecuador

E-mail: mlgonzalez@utmachala.edu.ec
} 


\begin{abstract}
Keywords:
Circular economy, export

Companies,

Competitive

advantage,

International

trade, Circular economy strategy of the European Union.

Abstract

Concern for environmental sustainability is becoming increasingly strong in the field of international business and government decisions. The "Circular Economy Strategy" is an example of a legislative initiative by the European Union, which reflects guidelines on the regulation of resource consumption and future relationships with its suppliers. The purpose of this research is to analyze the circular economy as a competitive strategy for exporting companies, based on the review of 15 scientific articles based on research from companies that have implemented the circular economy. The development of this research was based on a qualitative approach with the application of analytical-synthetic and historical-logical methods. The results indicate that companies are making changes in their production processes to help reduce the use of resources and improve waste management. It is concluded that $\mathrm{e}$ companies, at different rates, depending on their strengths and capacities, are managing to develop value proposals to underpin the sustainability of resources, managing to differentiate themselves from their competitors.
\end{abstract}

JEL Codes: F18, L21, M16

\section{INTRODUCCIÓN}

En las dos últimas décadas se ha destacado la presión que ejercen los consumidores frente a la difusión de las consecuencias ambientales por el uso inconsciente de los recursos (Elia, Gnoni y Tornese, 2017). Dicha presión está actuando como un impulsor para que las empresas decidan cambios en sus modelos comerciales pasadas.

En línea con lo anterior, las decisiones adoptadas por los gobiernos de los países más desarrollados reflejan sus objetivos de cara al consumo de los recursos y relaciones futuras con sus proveedores. Así, se destacan como ejemplos en Europa (Unión Europea [UE], 2020) la Ley de Cambio Climático y Transición Energética, la Agenda 2030, el Acuerdo de Paris relativo al cambio climático, el Plan de Acción de la Comisión Europea en relación con los lineamientos del Pacto Verde Europeo. Concretamente en España, "La Estrategia Española de Economía Circular", que supone pasos para la reducción del consumo de materiales, eficiencia en el uso del agua, reducción de residuos. Otro mercado importante como China dispone de la "Ley de Promoción de la Economía Circular de la República Popular China" (PRC, 2008), mientras Inglaterra posee la
Norma Británica 8001.2017, para orientar a las empresas sobre la implementación de los principios de EC (Pauliuk, 2017; Niero y Schmidt, 2018). Todas estas acciones están encaminadas a disminuir el efecto invernadero por las emisiones de gases vinculada al tratamiento de los residuos.

En este contexto, las empresas que participan en los mercados internacionales se enfrentan al reto de pasar de una Economía Lineal (EL) a los principios de la llamada Economía Circular (EC), como una estrategia para contribuir con la sociedad y el entorno a largo plazo. Consiste en la adquisición de materias primas, la elaboración de bienes, la utilización del producto y por último su desecho, generando residuos que afectan al medio ambiente y el consumo de los recursos (Arroyo, 2018). Bajo los principios de la EC, se promueve que los productos sean reutilizados $y$ no desechados en una primera instancia, rescatando de ellos las partes y piezas que aún sirven con el fin de reducir la contaminación (Ellen MacArthur Foundation, 2015; García, 2016).

Derivado de estos antecedentes, se distingue el creciente interés de las empresas por incorporar cambios en sus procesos, para reducir al mínimo 
el impacto en el medio ambiente (Niero y Schmidt, 2018). Las empresas buscan generar ventajas frente a sus competidores a partir de reflejar un comportamiento más responsable con la preservación de los recursos (Gaustad, Krystofik, Bustamante y Badami, 2018). De esta manera, las empresas que aplican la EC persiguen la integración de aspectos económicos, políticos, sociales y ambientales (Huysman, Schaepmeester, Ragaert, Dewulf y Meester, 2017).

En la revisión de la literatura se destacan investigaciones que impulsan la idea de que la aplicación de la EC ayuda a las empresas a crear una imagen atractiva para sus potenciales clientes e inversionistas, asociando sus operaciones a parámetros de sostenibilidad. Así, Kirchherr et al. (2017) en sus estudios concluyen que la EC contribuye principalmente a la prosperidad económica al desarrollo sostenible, siendo las empresas y los consumidores sus facilitadores. Geissdoerfer, Savaget, Bocken y Hultink (2017) señalan que existe un mayor interés en Europa y China por la EC, especialmente por parte de los empresarios y los responsables de formular políticas públicas. Por su parte, Prieto, Jaca y Ormazabal (2019) sostienen que las preferencias de los consumidores que manifiestan una mayor conciencia ambiental, se orientarán por sus percepciones sobre empresas que oferten productos y procesos sostenibles para su obtención. Otros autores como Pieroni, McAloone y Pigosso (2019) y Ünal, Urbinati y Chiaroni (2019) concuerdan en su criterio respecto que a medida que la EC se asocia a la sostenibilidad ambiental, y recibe una mayor atención por parte de los gobiernos y los consumidores, las empresas deben considerar la adopción de la EC para defender su ventaja competitiva.

Considerando que la adopción de la EC puede contribuir a mejorar las posibilidades de las empresas exportadoras, para ingresar y posicionarse en los mercados más exigentes y a la vez rentables, el presente trabajo se direcciona bajo la siguiente pregunta de investigación: ¿la adopción de la EC genera valor agregado a las empresas para competir en los mercados internacionales?, expuesta la interrogante, este artículo tiene por objetivo analizar la EC como estrategia competitiva para las empresas exportadoras. Para el cumplimiento del objetivo se utiliza un enfoque cualitativo, fundamentado en los métodos teóricos histórico-lógico y analítico-sintético. Se analizan 15 artículos científicos, los cuales contemplan el análisis de casos reales de empresas en varios sectores y origen geográfico.

La estructura del artículo comprende la introducción, seguido de la revisión de la literatura que expone una aproximación a la EC, sus implicaciones, referencias del marco normativo internacional. Se plantea la metodología, los resultados de los estudios revisados y su respectiva discusión. Finalmente se exponen las conclusiones, limitaciones y futuras líneas de investigación.

\section{MARCO TEÓRICO}

\section{Implicaciones de la Economía Circular}

El uso de recursos naturales y la gestión de residuos son en la actualidad un reto para las leyes y la sostenibilidad ambiental (Oblitas, Sangay, Rojas y Castro, 2019). Los empresarios tienen la responsabilidad de cuidar el medio ambiente, y los Estados deben impulsar políticas que favorezcan a las empresas en la adopción de medidas favorables para reducir la contaminación.

Martínez y Porcelli, (2019) indican que el vigente modelo de producción (lineal), ha ocasionado el desgaste de los recursos naturales, provocando la pérdida total de ecosistemas, con ello ha aumentado drásticamente la pobreza en las comunidades que dependen específicamente de los recursos que proporciona la naturaleza.

Las sociedades están impulsando la implementación de un modelo ecológicamente racional e inclusivo, que permita a las empresas reutilizar productos generando valor agregado, la llamada EC, que tiene como objetivo reemplazar el tradicional modelo de producción (Araujo, De Nadae, Clemente, Chinen y Monteiro, 2018). La EC está orientada a la modificación de hábitos de consumo, importancia de los recursos e 
incremento de vida útil de los productos, teniendo como resultado que las empresas que la apliquen sean sostenibles en el tiempo (Moscoso, Rojas y Beraún, 2019). Así, Ritzén y Ölundh, (2017) sugieren la utilización de los residuos en lugar de desecharlos, permitiendo la reducción del uso de recursos y energía.

Al resolver los problemas medioambientales, se crean oportunidades de negocio y crecimiento económico para las empresas dedicadas a la fabricación e innovación de productos, siendo la innovación uno de los factores más importantes para la inserción a la economía circular. La EC exige cambios en la cadena de valor, comenzando por el diseño, nuevos modelos para el mercado, gestión y transformación de los residuos en generadores de activos, hasta el análisis de la conducta a los consumidores (Melgarejo, 2019). En este sentido, Vence y Pereira, (2018) señalan que la eco-innovación para la EC puede ser tecnológica y no tecnológica, de hecho, aborda temas como los modelos económicos y leyes.

En la tabla 1, encontrada en apendice se sintetizan las argumentaciones de varios autores sobre lo que implica la EC, para aproximarnos a una mejor comprensión de las prácticas y su contexto.

De acuerdo a las definiciones presentadas en el aprendice, tabla 1, la EC se enfoca en el uso de energías renovables, eficiencia energética, gestión eficiente y responsabilidad sobre los recursos renovables y no renovables (Espaliat, 2017). A continuación se resumen consideraciones a manera de principios de la EC.

\section{Principios de la Economía Circular}

1. Disminución de desechos en la elaboración de nuevos productos mediante la mejora de los ciclos de producción, otorgándole una segunda vida útil al producto ya utilizado, añadiéndole valor.

2. Los productos y servicios deben ser creados para diversas utilizaciones en todo su ciclo de vida.

3. Utilización únicamente de recursos renovables, ya que su disponibilidad es ilimitada, para así reducir en impacto negativo medioambiental.

4. Los desechos orgánicos no son basura y se pueden transformar en recurso importante para los ciclos biológicos.

5. Aumentar el valor de las materias primas.

\section{Referencias normativas de la Economía Circular}

$\mathrm{Si}$ bien los consumidores son los agentes económicos principales para el impulso de la EC, en la actualidad se destacan los esfuerzos de la Unión Europea en el diseño de un marco normativo, que rige para un mercado integrado. Los países que forman parte de la Unión Europea deben cumplir con una serie de compromisos, en función de su propia normativa estatal, la cual está relacionada a los Objetivos del Milenio, Agenda 2030 y el Acuerdo de Paris, que en esencia apuntan a mitigar el cambio climático.

De acuerdo con la Comisión Europea (2015) existen indicaciones claras para la sociedad, respecto a la gestión de residuos que vincula, en primera instancia a las administraciones municipales. No obstante, el objetivo primordial de la EC, es la reducción de residuos, que involucra a las empresas que ponen a disposición del público consumidor de sus productos. De ahí que las normativas sobre EC establecidas en el gran mercado de la Unión Europea, sirvan como alertas para las acciones futuras de las empresas exportadoras.

En este sentido, la EC se relaciona con un cambio sistémico, que integra a la sociedad consumidora y la gestión de las empresas que deben adoptar modificaciones en sus sistemas de producción, innovando el diseño de procesos y productos, orientada al eco-diseño, envases reutilizables, de uso múltiple, reducción del uso de plásticos.

La Unión Europea monitorea el cumplimiento de sus miembros sobre los objetivos en el marco de la EC, específicamente a través de la Directiva Marco de Residuos 2008/98/CE, modificada por la Directiva 2018/851, la cual acompañada de un grupo de reglamentos, Decisiones y Directivas, 
robustece el camino hacia la EC y el cambio climático (De la Vega, 2019). Cabe destacar que entre este grupo normativo existen incentivos económicos centrados en los productores, para impulsar el diseño de productos ecológicos que contribuyan al reciclaje de envases, por ejemplo. Así, el reciclaje actúa como la función más visible del EC, mientras la Unión Europea manifiesta sus acciones por convertirse en una sociedad que valora y practica el reciclaje como una forma de adaptarse el cambio climático.

Estas iniciativas permiten brindar respuesta a la emergencia climática, mediante actuaciones coherentes donde la prioridad no es el mercado sino la sostenibilidad del medio ambiente para futuras generaciones. Los sectores prioritarios en los que se enfoca la Directiva 2018/851 de la Unión Europea, se refieren a materias primas críticas, construcción y demolición, biomasa y bioproductos. Mención especial merece el caso de plásticos y residuos alimentarios que tienen una mayor relación con las exportaciones latinoamericanas a la Unión Europea.

Se destacan las acciones de España en donde existen varias normativas autonómicas sobre el cambio climático y la transición hacia la EC, así la ley de Cataluña, Andalucía, de las Islas Baleares, la ley de Navarra. Estas iniciativas se refuerzan con la llamada Estrategia Española de Economía Circular, que marca referencias para superar la EL y posicionar la EC como un nuevo modelo de producción y consumo.

En Apéndice tabla 2 se recoge, a manera de referencia, una breve descripción del alcance de medidas incorporadas en el Plan de acción de la Unión Europea para la Economía Circular.

En la revisión de literatura, De la Vega (2019) se puede observar que los objetivos de la Unión Europea en materia de EC están definidos, y entre sus miembros más activos al difundir las estrategias de desarrollo de la EC se encuentran: Alemania, Bélgica, España, Francia, Holanda y Hungría, por otro lado, fuera de la Unión Europea, Irlanda y Reino Unido.

\section{METODOLOGÍA}

Con el objetivo trazado que consiste en analizar la economía circular como estrategia competitiva para las empresas exportadoras, para esta investigación se aplica un enfoque cualitativo. De acuerdo con Hernández, Fernández y Baptista (2014), el enfoque cualitativo es aquel que hace uso de la información recopilada en otras investigaciones, es decir, utiliza la recolección e interpretación de datos de otros autores para tomar decisiones sobre un tema en específico.

Se aplica el método histórico-lógico y analíticosintético, denominados métodos teóricos, empleados para analizar e interpretar el planteamiento de diferentes autores seleccionados para la revisión de la literatura.

Para el desarrollo de esta investigación, se obtuvo información de publicaciones de prestigiosas revistas científicas, como Journal of Cleaner Production, Contaduría y Administración, Cuadernos de Prospecção, Revista Chilena de Relaciones Internacionales, Revista de Estudios Europeos, Revista Brasileira de Ciências Ambientais, Revista Catalana de DRET Ambiental, además, se indagó en páginas web como Google Académico y sitios oficiales de la Unión europea de donde se extrajo documentos.

Para la selección de revisión de la literatura en las fuentes de investigación (revistas científicas) se usó palabras de motores de búsqueda como "economía circular", "exportadoras y economía circular", "ecología industrial", "circular economy", "residuos". En esta investigación solo se incluyen publicaciones comprendidas en los últimos 5 años.

Para los resultados se realizó un análisis de 15 publicaciones, las cuales contemplan casos de expresas exportadoras de diversos sectores económicos que adoptan la EC en sus procesos de producción.

\section{RESULTADOS}

En los mercados cambiantes, las empresas buscan defender su competitividad y permanencia. Por lo tanto, adoptan nuevas estrategias, prácticas organizacionales y 
medioambientales, para defenderse y hacer frente a los cambios que se generan día a día.

En Apéndice tabla 3, se recogen 15 estudios precedentes elaborados por otros autores, los mismos que exponen casos de empresas orientadas al mercado internacional que adoptaron la EC en sus procesos de producción como una estrategia.

En los resultados obtenidos se reflejan las experiencias de empresas de diferentes países, que para ser amigables con el medio ambiente han adoptado en sus procesos de producción la EC, para con ello, generar ventajas competitivas en comparación con las demás empresas que aún operan bajo una economía lineal. De los casos abordados se destaca, que la aplicación de la EC se enfoca en procesos de producción, servicios, producto y organización. Las empresas buscan disminución de gastos en la cadena de producción y reducción del impacto ambiental.

En algunos casos las empresas se encuentran aún en la primera fase de implementación de las prácticas medioambientales, por lo que es necesario, la adquisición de mayor conocimiento sobre los principios de la EC, políticas y prácticas, gestión de residuos, además, implementar estrategias para la correcta aplicación de la EC (Jørgensena y Remmen, 2019). En concordancia con Ritzén y Ölundh (2017), se observa que la inserción de las empresas en el modelo económico de EC es un desafío, ya que implica más funciones, debido a los nuevos procedimientos y estructuras en cuanto a la innovación.

En línea con los resultados de otros estudios (Espaliat, 2017; Ritzén y Ölundh, 2017), los hallazgos de la presente investigación permiten reforzar la idea de que la aplicación de la EC trae consigo ciertas ventajas, las cuales se sintetizan a continuación:

1. Crecimiento económico: obtenido mediante la combinación de ingresos derivados de las actividades emergentes y la disminución de los costos de producción al utilizar de manera más productiva los insumos.
2. Ahorro en el costo de materia prima: mediante la reutilización de productos cuya principal utilización ha llegado a su fin, y serán reparados, reelaborados o desmontado sus piezas principales, produciendo ahorro en los sistemas de producción.

3. Creación de valor: el aumento en la productividad genera un impacto positivo.

4. Reputación: debido a la concienciación por parte de los consumidores, el cuidado del medio ambiente o responsabilidad social ha tomado un papel fundamental en los modelos de negocios, y la adpción de la EC en las empresas es garantizar la reputación de la misma.

5. Competitividad: la economía circular se basa en tecnología e implantación de procesos innovadores lo que garantiza la competencia.

Los hallazgos permiten sostener que la $\mathrm{EC}$ es de gran importancia para las empresas que participan en los mercados internacionales, debido a que su implementación conlleva a que las empresas exportadoras se muestren más atractivas en términos comerciales, en concordancia con la visión de Geissdoerfer et al. (2017); Kirchherr et al. (2017); Gaustad et al. (2018); Melgarejo (2019) sobre los efectos de la EC en la competitividad global de las empresas.

\section{CONCLUSIONES}

En el escenario de los mercados internacionales, los actores sociales y representantes de gobiernos vienen demostrando su interés por acciones concretas para frenar el cambio climático, siendo una realidad la Estrategia de Economía Circular por parte de la Unión Europea. Bajo esta premisa, el presente trabajo se ha guiado por el objetivo de analizar la Economía Circular como estrategia competitiva para las empresas exportadoras.

Una mayor conciencia de los consumidores por aspectos ambientales es reforzada por la legislación europea, ante lo cual, las empresas exportadoras deben hacer una lectura obligada de las reglas del mercado, que afectan concretamente 
a los procesos de producción y consumo. Las modificaciones en el ámbito normativo podrían ser apreciadas por las empresas exportadoras como oportunidades para contribuir al cambio de modelo económico, y no necesariamente como una amenaza por las exigencias derivadas en la gestión de residuos y reciclaje. La adopción de los principios de Economía Circular, como respuesta estratégica, permitiría a las empresas exportadoras aumentar sus posibilidades de competir en escenarios complejos que exigen el abordaje de la sostenibilidad de los recursos, cada vez más presente en las necesidades de los principales mercados internacionales.

El análisis de 15 artículos científicos que han tratado sobre la experiencia de empresas en la aplicación de la Economía Circular ha permitido dar soporte a los resultados y abordar la interrogante de investigación planteada: ¿la adopción de la Economía Circular genera valor agregado a las empresas para competir en los mercados internacionales? Al hilo de lo expuesto, se ha identificado que, a partir de innovaciones organizativas, asociadas a los principios de Economía Circular, las empresas de diversos sectores y ubicación geográfica han modificado sus procesos productivos, mejorando el diseño de productos, la reutilización y minimización del uso de los recursos, la gestión de residuos, la gestión eficiente del agua y el uso de energías renovables.

En el ámbito de los hallazgos de referencia, se concluye que las empresas exportadoras aplican la Economía Circular con diferentes ritmos y niveles en función de sus fortalezas y debilidades, reflejadas en parte por su capacidad financiera, operativa y organizacional (Gaustad et al., 2018; Niero y Schmidt, 2018). Finalmente, las empresas están consiguiendo disminuir el uso de recursos, mediante el desarrollo de propuestas de valor para apuntalar la sostenibilidad de los recursos, logrando diferenciarse de sus competidores.

La presente investigación como una aproximación a la Economía Circular puede abonar al análisis estratégico externo de las empresas que requieren dimensionar los cambios del mercado para responder de forma adecuada. Por otro lado, considerando como principal limitación el enfoque cualitativo en los temas tratados, las futuras líneas de investigación podrían abordar las barreras e incertidumbres que pesan sobre la implementación de la Economía Circular, desde un enfoque cuantitativo que permita recoger percepción de los empresarios de cara a los retos que involucra la superación el modelo de Economía Lineal.

\section{REFERENCIAS}

Araujo, G., De Nadae, J., Clemente, D., Chinen, G., \& Monteiro, M. (2018). Circular Economy: Overview of Barriers. Procedia CIRP, 73, 79-85. doi:https://doi.org/10.1016/j.procir.2018.04.011

Arroyo, F. (2018). La economía circular como factor de desarrollo sustentable del sector productivo. INNOVA Research Journal, 3(12), 78-98.

doi:https://doi.org/10.33890/innova.v3.n12.2018. 786

Baldassarre, B., Schepers, M., Bocken, N., Cuppen, E., Korevaar, G., \& Calabretta, G. (2019). Industrial Symbiosis: towards a design process for eco-industrial clusters by integrating Circular Economy and Industrial Ecology perspectives. 216, 446-460. doi:https://doi.org/10.1016/j.jclepro.2019.01.091

Bassi, F., \& Dias, J. (2019). The use of circular economy practices in SMEs across the EU. Resources, Conservation \& Recycling, 144. doi:https://doi.org/10.1016/j.resconrec.2019.03.0 19

Blomsma, F., \& Tennant, M. (2020). Circular economy: Preserving materials or products? Introducing the Resource States framework. Resources, Conservation \& Recycling, 156, 1-13. doi:https://doi.org/10.1016/j.resconrec.2020.104 698

Comisión Europea. (2014). Comunicación de la Comisión al Parlamento Europeo, al concejo, al comité económico y social Europeo y al comité de las regiones "Hacia una economía circular: un programa de cero residuos para Europa". Bruselas: COM(2014) 398 final. 
Comisión Europea. (2015). Comunicación de la Comisión al Parlamento Europeo, al consejo, al comité económico y social Europeo y al comité de las regiones "Cerrar el círculo: un plan de acción de la UE para la economía circular". Bruselas: $\operatorname{COM}(2015)$.

Comisión Europea. (11 de Marzo de 2020). Modificar nuestras pautas de producción y consumo: El nuevo Plan de acción para la economía circular muestra el camino hacia una economía competitiva y climáticamente neutra de consumidores empoderados. [Comunicado de prensa]. Bruselas. Obtenido de https://ec.europa.eu/commission/presscorner/deta il/es/ip_20_420

Comisión Europea. (2020). Plan de accion para una economía circular. EU Green Deal Circular Economía. doi:doi:10.2775/4448

Córdova, M. (13 de Mayo de 2019). repositorio.tec. Obtenido de https://repositorio.tec.mx/handle/11285/633054

De la Vega, P. (del 10 al 11 de octubre de 2019). La incorporación de la econoía circular de la legislación estatal de residuos a raíz de la directiva (UE) 2018/851 (ponencia). VIII Congreso Nacional de Derecho Ambiental. Obtenido de https://www.datocmsassets.com/28023/1597007393-actualidadjuridica-ambientalcomercio-internacional-yderecho-ambiental.pdf

Elia, V., Gnoni, M., \& Tornese, F. (2017). Measuring circular economy strategies through index methods: A critical analysis. Journal of Cleaner Production, 142, 2741- 2751. doi:http://dx.doi.org/10.1016/j.jclepro.2016.10.1 96

Ellen MacArthur Foundation. (2015). Towards the Circular Economy. Obtenido de https://www.ellenmacarthurfoundation.org/assets /downloads/publications/Executive_summary_S P.pdf

Espaliat, M. (2017). Economía Ciruclar y Sostenibilidad: Nuevos enfoques para la creación de valor (Primera ed.). Chile: CreateSpace
Independent Publishing Platform. Obtenido de https://wolfypablo.com/documentacion/documen tos/2017-

10/710\%20Economia_circular_y_sostenibilidad. pdf

Gall, M., Wiener, M., Oliveira, C. C., Lang, R., \& Hansenb, E. (2020). Building a circular plastics economy with informal waste pickers: Recyclate quality, business model, and societal impacts. Recursos, conservación y reciclaje, 156, 104685. doi:https://doi.org/10.1016/j.resconrec.2020.104 685

García, S. (2016). Economía Circular: La Unión Europea impulsa reformas sobre la base de un tema crucial, la gestión de residuos, con el fin de alcanzar mejoras económicas y medioambientales. Actualidad Jurídica Ambiental(57), 1-11. Obtenido de https://dialnet.unirioja.es/servlet/articulo?codigo $=5704204$

García, S. (2018). Economía Circular: 30 años del principio del desarrollo sostenible evolucionan en el nuevo gran objetivo medioambiental de la Unión Europea. Revista de Estudios Europeos(71), 309-321. Obtenido de http://www.ree-uva.es/

Gaustad, G., Krystofik, M., Bustamante, M., \& Badami, K. (2018). Circular economy strategies for mitigating critical material supply issues. Resources, Conservation and Recycling(135), 2433.

doi:https://doi.org/10.1016/j.resconrec.2017.08.0 02

Geissdoerfer, M., Savaget, P., Bocken, N., \& Hultink, E. (2017). La economía circular: ¿un nuevo paradigma de sostenibilidad? Revista de producción más limpia, 757-768. doi:https://doi.org/10.1016/j.jclepro.2016.12.048

González, J., Ovalle, M., \& Salazar, M. (2018). La Economía Circular como respuesta alternativa a los desafios de la alimentación: análisis de caso para la situación de Chile. Revista Chilena de Relaciones Internacionales., 2(2), 94-104. 
Hernández, R., Fernández, C., \& Baptista, M. (2014). Metodología dela investigación (Sexta ed.). México: McGraw-Hill. Obtenido de https://periodicooficial.jalisco.gob.mx/sites/perio dicooficial.jalisco.gob.mx/files/metodologia_de_ la_investigacion_-

_roberto_hernandez_sampieri.pdf

Huysman, S., Schaepmeester, J. D., Ragaert, K., Dewulf, J., \& Meester, S. D. (2017). Performance indicators for a circular economy: A case study on post-industrial plastic waste. Resources, Conservation and Recycling, 120, 46-54. doi:http://dx.doi.org/10.1016/j.resconrec.2017.01 .013

Jørgensena, M., \& Remmen, A. (2019). methodological approach to development of circular economy options in businesses. $\begin{array}{lllll}\text { ScienceDirect, } & 69, & 816 & - & 821 .\end{array}$ doi:https://doi:10.1016/j.procir.2017.12.002

Kirchherr, J., Piscicelli, L., Bour, R., Kostense, E., Muller, J., Huibrechtse, A., \& Hekkert, M. (2018). Barriers to the Circular Economy: Evidence from the European Union (EU). Ecological Economics, 150, 264-272. doi:https://doi.org/10.1016/j.ecolecon.2018.04.0 28

Kowszyk, Y., \& Maher, R. (2018). Estudios de caso sobre modelos de Economía Circular e integración de los Objetivos de Desarrollo Sostenible en estrategias empresariales en la UE y ALC. Alemania: Fundación EU-LAC. doi:10.12858.1018ES

Marcet, X., Marcet, M., \& Vergés, F. (2018). Qué es la economía circular y por qué es importante para el territorio. Barcelona: Asociación Pacto Industrial de la Región Metropolitana de Barcelona. Obtenido de www.pacteindustrial.org.

Martínez, A., \& Porcelli, A. (2019). Estudio sobre la economía circular como una alternativa sustentable frente al ocaso de la economía tradicional. Lex, 17(23), 1-40. doi:http://dx.doi.org/10.21503/lex.v17i23.1679

Melgarejo, V. (2019). Economía Circular y la Industria Textil en el Paraguay. Población y
Desarrollo, 25(49), 143-150. doi:http://dx.doi.org/10.18004/pdfce/2076-054x

Moneva, J., Portillo, P., Llena, F., \& Scarpellini, S. (2018). Perspectivas e impacto de la Economia Circular en Aragón desde la optica empresarial. Ecnomía Argonesa, 111-126.

Morán, P. (Septiembre de 2019). Repositorio Universidad de Guayaquil. Obtenido de http://repositorio.ug.edu.ec/handle/redug/45807

Morató, J., Tollin, N., \& Jiménez, L. (2017). Situación y evolución de la Economía Circular en España. Madrid: Fundación COTEC para la Innovación.

Moscoso, K., Rojas, C., \& Beraún, M. (2019). La economía circular: modelo de gestión de calidad en el Perú. Revista de Investigación $P U R I Q$ UNAH-HUANTA, 1(2), 189-202. doi:https://doi.org/10.37073/puriq.1.02.48

Niero, M., \& Schmidt, X. (2018). The Role of Life Cycle Sustainability Assessment in the Implementation of Circular Economy Principles in Organizations. ScienceDirect, 69, 793 - 798. doi:https://doi.org/10.1016/j.procir.2017.11.022

Oblitas, J., Sangay, M., Rojas, E., \& Castro, W. (2019). Economía circular en residuos de aparatos eléctricos y electrónicos. Revista de Ciencias Sociales (Ve), 25(4), 1-11. Obtenido de https://www.redalyc.org/jatsRepo/280/28062322 016/html/index.html

Pauliuk, S. (2017). Critical appraisal of the circular economy standard BS 8001:2017 and a dashboard of quantitative system indicators for its implementation in organizations. Resources, Conservation and Recycling, 129, 81-92. doi:https://doi.org/10.1016/j.resconrec.2017.10.0 19

Pieroni, M., McAloone, T., \& Pigosso, D. (2019). Business model innovation for circular economy and sustainability: A review of approaches. Journal of Cleaner Production, 215, 198-216. doi:https://doi.org/10.1016/j.jclepro.2019.01.036 
Prieto, V., Jaca, C., \& Ormazabal, M. (2019). Towards a consensus on the circular economy. Journal of Cleaner Production(179), 605-615. doi:https://doi.org/10.1016/j.jclepro.2017.12.224

Ritzén, S., \& Ölundh, G. (2017). Barriers to the Circular Economy - integration of perspectives and domains. Procedia CIRP, 64, 7-12. doi:https://doi.org/10.1016/j.procir.2017.03.005

Tabares, A., \& Borschiver, S. (2020). Contribuições do Segmento de Produtos de Limpeza para a Economia Circular. Cuadernos de Prospecção, 13, 974-988. doi:https://doi:10.9771/cp.v13i4.32743

Ünal, E., \& Shao, J. (2018). A Taxonomy of Circular Economy Implementation Strategies for Manufacturing Firms: Analysis of 391 Cradle-toCradle Products. Journal of Cleaner Production, 212, 754-765. doi:10.1016/j.jclepro.2018.11.291

Ünal, E., Urbinati, A., \& Chiaroni, D. (2019). Prácticas de gestión para el diseño de modelos de negocio de economía circular: el caso de una PYME italiana en la industria de suministros de oficina. Journal of Manufacturing Technology Management, $\quad 30(3), \quad 516-589$. doi:https://doi.org/10.1108/JMTM-02-2018-0061

Vence, X., \& Pereira, Á. (2018). Eco-innovation and Circular Business Models as drivers for a circular economy. Contaduría y Administración, 64(1), 1-19. doi:http://dx.doi.org/10.22201/fca.24488

Werning, J. P., \& Spinler, S. (2019). Transition to Circular Economy on Firm Level: Barrier identification and prioritization along the value chain. Journal of Cleaner Production, 245, 118609.

doi:ttps://doi.org/10.1016/j.jclepro.2019.118609

Zabaleta, V. (2020). repositorio.unbosque. Obtenido de https://repositorio.unbosque.edu.co/bitstream/ha ndle/20.500.12495/3041/Zabaleta_Guzman_Vale ntina_2020.pdf? sequence $=1 \&$ isAllowed $=y$ 


\section{APÉNDICES \\ TABLA 1 \\ Aproximaciones teóricas a la Economía Circular.}

\begin{tabular}{|c|c|}
\hline Autores & Aportes sobre Economía Circular \\
\hline $\begin{array}{l}\text { Comisión Europea } \\
\text { (2014) }\end{array}$ & $\begin{array}{l}\text { Comprende la inclusión del material residual, maximizando su vida útil. Su objetivo } \\
\text { es mantener los recursos dentro de la economía, aun cuando el tiempo de vida útil } \\
\text { del producto ha llegado a su fase final, de esta forma se continúa utilizándolo } \\
\text { generando valor. }\end{array}$ \\
\hline $\begin{array}{l}\text { Ellen MacArthur } \\
\text { Foundation (2015) }\end{array}$ & $\begin{array}{l}\text { Es el denominado sistema industrial restaurativo y reemplaza el concepto "fin de } \\
\text { vida útil", haciendo posible el uso de energía renovable, eliminación de desechos } \\
\text { tóxicos que afectan al medio ambiente. }\end{array}$ \\
\hline García (2016) & $\begin{array}{l}\text { Mantiene por mayor tiempo el valor agregado de los productos y descarta los } \\
\text { residuos. De este modo, no solo se protege al medio ambiente, sino que también se } \\
\text { mantendría y aumentaría las plazas de trabajo, innovación y más adelante generaría } \\
\text { ventajas competitivas a las empresas. }\end{array}$ \\
\hline $\begin{array}{l}\text { Morató, Tollin y } \\
\text { Jiménez (2017) }\end{array}$ & $\begin{array}{l}\text { Contempla la innovación de los sistemas de producción y consumo, procurando el } \\
\text { desarrollo socioeconómico, aportando a la creación de fuentes de trabajo y } \\
\text { conservando los recursos. }\end{array}$ \\
\hline García (2018) & $\begin{array}{l}\text { Implementación de nuevos modelos económicos donde se utilice materiales } \\
\text { residuales para su reutilizaciones, lo que se llama actualmente residuos, de esta } \\
\text { menera se protege el medio ambiente. }\end{array}$ \\
\hline $\begin{array}{l}\text { González, Ovalle } \\
\text { y Salazar (2018) }\end{array}$ & $\begin{array}{l}\text { La EC propone la utilización de energia renovabe para la fabricación y diseño de } \\
\text { nuevos productos, que sean reusables y totalmente reciclables, por lo tanto, dejan } \\
\text { de existir los desechos. }\end{array}$ \\
\hline $\begin{array}{l}\text { Kowszyk y Maher } \\
(2018)\end{array}$ & $\begin{array}{l}\text { Estrategia alternativa al modelo lineal, la economía circular reduce la } \\
\text { contaminación ambiental, en el cual el bien o servicio final es la fuente de valor. Su } \\
\text { objetivo es maximizar la vida útil del producto. }\end{array}$ \\
\hline $\begin{array}{l}\text { Marcet, Marcet } \\
\text { y Vergés (2018) }\end{array}$ & $\begin{array}{l}\text { Los bienes y procesos deben ser planteados pensando en el sistema circular, en otras } \\
\text { palabras, para la elaboración de productos se debe tener en cuenta que los materiales } \\
\text { utilizados no pierdan su valor al momento de su reinserción al sistema productivo, } \\
\text { para maximizar su vida útil o mejorarla. }\end{array}$ \\
\hline
\end{tabular}

Fuente: Elaboración propia a partir de los autores citados. 


\section{Tabla 2}

Plan de acción de la Unión Europea para la Economía Circular

\begin{tabular}{|c|c|}
\hline Acciones prioritarias & Finalidad \\
\hline Consumo & $\begin{array}{l}\text { Empoderar a los consumidores con el fin de concientizar sobre el } \\
\text { cuidado y protección del medio ambiente. Promover que los } \\
\text { productos sostenibles sean la norma de la UE. Los productos } \\
\text { comercializados deben ser reutilizables, además de restringir } \\
\text { productos de una sola vida útil. }\end{array}$ \\
\hline Diseño y producción & $\begin{array}{l}\text { Implantación de un plan de trabajo sobre diseño ecológico, que } \\
\text { incluya normas sobre los requisitos de eficiencia de los materiales, } \\
\text { entre otros. Implementación del European Circular Economy } \\
\text { Stakeholder Platform, sitio web que promueve la aplicación de la } \\
\text { economía circular, brindando información a las empresas interesadas. }\end{array}$ \\
\hline Gestión de residuos & $\begin{array}{l}\text { Principio de jerarquía de residuos para fomentar el reciclado y la } \\
\text { reutilización. Reducción de plásticos, promover que los envases de } \\
\text { plástico sean reciclables. Prevenir en las empresas el exceso de } \\
\text { embalaje en ciertos productos. }\end{array}$ \\
\hline Producción textil & Impulsar la reutilización textil en el mercado europeo. \\
\hline $\begin{array}{l}\text { Implantación de } \\
\text { sistemas de gestión } \\
\text { ambiental }\end{array}$ & $\begin{array}{l}\text { Para incrementar la eficiencia e innovación de los procesos } \\
\text { productivos. Fomentar el uso de indicadores que permitan conocer el } \\
\text { grado de implementación de EC. }\end{array}$ \\
\hline $\begin{array}{l}\text { Inversión } \\
\text { innovación }\end{array}$ & $\begin{array}{l}\text { Financiar proyectos innovadores en proyectos referentes a economía } \\
\text { circular. }\end{array}$ \\
\hline $\begin{array}{l}\text { Gestión eficiente del } \\
\text { agua }\end{array}$ & $\begin{array}{l}\text { Reducir el mal uso del líquido vital, además de fomentar su } \\
\text { reutilización. }\end{array}$ \\
\hline
\end{tabular}

Fuente: Elaboración propia a partir de Comisión Europea (2020) 
Tabla 3

Casos de empresas que realizan prácticas en el marco de la Economía Circular

\begin{tabular}{|c|c|c|c|}
\hline $\begin{array}{c}\text { AUTOR } \\
\text { Ã̃NO }\end{array}$ & & MUESTRA & PRINCIPALES RESULTADOS \\
\hline $\begin{array}{l}\text { Tabares } \\
\text { Borschiver } \\
(2020)\end{array}$ & & $\begin{array}{l}3 \text { empresas, sector } \\
\text { industrial, Estados } \\
\text { Unidos. }\end{array}$ & $\begin{array}{l}\text { Grandes avances en la aplicación de los principios de la EC, } \\
\text { además, se demostró un desarrollo en la fabricación del } \\
\text { producto detergente, su elaboración es a base de encimas, y } \\
\text { sustituyeron los envases plásticos por bolsas recargables, lo } \\
\text { que reflejó resultados positivos, en cuanto al ahorro de } \\
\text { plástico y agua. }\end{array}$ \\
\hline $\begin{array}{l}\text { Moneva, } \\
\text { Portillo, } \\
\text { Llena } \\
\text { Scarpellini } \\
(2018)\end{array}$ & $\mathrm{y}$ & $\begin{array}{l}51 \text { empresas, } \\
\text { sectores } \\
\text { alimentación, } \\
\text { residuos, } \\
\text { manufactura, } \\
\text { industrial, } \\
\text { transporte/logística, } \\
\text { sector servicios, } \\
\text { España-Aragón. }\end{array}$ & $\begin{array}{l}\text { En las empresas estudiadas se pudo determinar que la } \\
\text { aplicación de la EC se encuentra en primera fase, entre las } \\
\text { actividades que realizan están: eco-diseño de los productos, } \\
\text { cambios en los procesos de producción para que los } \\
\text { productos tengan mayor durabilidad. }\end{array}$ \\
\hline $\begin{array}{l}\text { Jørgensena } \\
\text { Remmen } \\
(2019)\end{array}$ & $\mathrm{y}$ & $\begin{array}{l}20 \text { empresas, sector } \\
\text { productivo } \\
\text { servicios, } \\
\text { Dinamarca. }\end{array}$ & $\begin{array}{l}\text { Las empresas aplicaron un modelo de negocio en la que se } \\
\text { cambió un producto de un solo uso por un producto } \\
\text { reutilizable, utilizaron } 3 \text { procesos de rediseño: rediseño } \\
\text { proporcionado (ofertas comerciales para retirar el producto } \\
\text { que el cliente bota después del uso), rediseño en la cadena } \\
\text { de valor ( propusieron descuentos en la siguiente compra si } \\
\text { el cliente devuelve el producto usado en lugar de botarlo) y } \\
\text { rediseño de interno (emplearon nuevas actividades como: } \\
\text { desmontaje, limpieza e producto usado, renovación, etc., } \\
\text { además dar información sobre la calidad de los productos } \\
\text { reutilizados). Estos tres rediseños hicieron posible el cambio } \\
\text { del producto. }\end{array}$ \\
\hline $\begin{array}{l}\text { Huysman } \\
\text { al. (2017) }\end{array}$ & & $\begin{array}{l}5 \text { empresas, sector } \\
\text { industrial, Bélgica e } \\
\text { Inglaterra. }\end{array}$ & $\begin{array}{l}\text { El resultado de las empresas estudiada, muestran que el } \\
\text { indicador de rendimiento de la EC, es una guía útil para que } \\
\text { el flujo de residuos tengan un valor óptimo, consiguiendo de } \\
\text { este modo proteger el medio ambiente. } \\
\text { La empresa A, es productora de películas de plástico, por lo } \\
\text { tanto, genera residuos de polietileno (PE). Los residuos más } \\
\text { puros son transportados a la empresa B (productora de } \\
\text { películas de plástico) y sometidos a reciclaje de circuito } \\
\text { cerrado. Los residuos sobrantes con pequeñas cantidades de } \\
\text { impurezas son transportadas a la empresa C, que es } \\
\text { productora de bolsas de residuos, estos residuos son } \\
\text { sometidos a un reciclado, lo sobrante es transportado a la } \\
\text { empresa D, para un reciclado en circuito abierto, por último, } \\
\text { la empresa E, realiza la incineración con recuperación de } \\
\text { energía. }\end{array}$ \\
\hline
\end{tabular}




$\begin{array}{lll}\text { Bassi y Dias } & 10.618 & \text { PYMES, } \\ \text { (2019) } & \begin{array}{l}\text { sector fabricación, } \\ \text { venta minorista y }\end{array} & \text { realizan por lo menos } 1 \text { actividad verde entre: re-planeación } \\ \text { servicios, Unión } & \text { maximizar su reutilización, volver a revisar las acciones en } \\ \text { Europea. } & & \begin{array}{l}\text { el uso de energía para minimizar su consumo, reducir los } \\ \text { residuos a través del reciclaje o reutilización, nuevos diseños }\end{array} \\ & & \text { a los productos y servicios para de este modo utilizar } \\ & \text { materiales reciclados o minimizar el uso de materiales. }\end{array}$

Gaustad et al. 1 empresa. Sector (2018) industrial, Estados Unidos.

La empresa estudiada aplica un enfoque de revertir en la cual involucra un cambio mínimo en sus procesos de fundición, para así poder reutilizar los desechos mediante fundición y agregarla masa maestra; recuperar; reciclar, identificar las piezas y limpiarlas para luego fundirlas y utilizarlas en la mezcla maestra. Con ello obtuvieron beneficios económicos como el ahorro, responsabilidad social corporativa mejorada.

$\begin{array}{lll}\text { Niero } & \text { y } & 2 \text { empresas, sector } \\ \text { Schmidt } & \text { industrial y de } \\ \text { (2018) } & \text { servicios, Reino } \\ & \text { Unido. }\end{array}$

Los estudios revelaron que las empresas adoptaron la norma BS 8001: 2017 y BS8001: 2017 - LCSA, respectivamente, las cuales, son normas y/o guías para la aplicación de EC. Estas normas pueden ser aplicadas en cualquier modelo de negocio, con el fin de generar competitividad ante los competidores siendo amigables con el medio ambiente.

Ünal y Shao 187 empresas, La implementación de estrategias para la aplicación de (2018) sector economía circular ha ayudado a las empresas manufacturero, manufactureras ser competitivas. Entre las estrategias China. adoptadas: reutilización de los materiales, utilización de energía renovable, administración de agua y equidad social, los resultados muestran que cada empresa aplica una estrategia diferente.

Blomsma y 1 empresa, sector La empresa analizada rediseño el producto mediante Tennant industrial, estrategias circulares que se definieron para abordar los (2020) desechos. Esto permitió la Re-manufacturación del producto, cambio rápido y fácil de piezas.

Gall, Wiener, 1 empresa, sector Oliveira, manufacturero, Lang y Nairobi.

Hansenb

El resultado de estudio permitió identificar que la empresa se dedica al comercio y procesamiento de productos postconsumo. Además, aplica la EC, ya que la empresa compra materiales reciclados y abarca la idea de que los recicladores son "héroes invisibles" de la gestión de residuos, debido a las contribuciones positivas que general al medio ambiente. Abandonó la cadena de valor tradicional y la reemplazó por una relación directa de comercio justo entre el reciclador individual y el reciclador empresa. Capacitaron a los recicladores para saber qué tipo de residuo plástico recolectar.

$\begin{array}{llll}\begin{array}{l}\text { Werning } \\ \text { Spinler }\end{array} & \text { y } & \begin{array}{l}1 \text { empresa, sector } \\ \text { tecnológico, Japón. }\end{array} & \begin{array}{l}\text { Para la aplicación de la economía circular, ha superado } \\ \text { barreras como la organización de la logística continua, Re- } \\ \text { manufacturación eficiente, alinear los objetivos de venta de } \\ \text { los nuevos productos, crear una marca para satisfacer las } \\ \text { necesidades del nuevo mercado. }\end{array}\end{array}$


Otra de la barrera superada esta relacionada con la percepción de productos remanufacturados como "de segunda mano" y, por tanto, de menor calidad, abordaron esta barrera al cambiar la marca de sus productos remanufacturados como productos "GreenLine" con un diferente enfoque de mercado y cliente.

Baldassarre et 1 empresa, sector En el caso de estudio, se determina que el tipo de sistema al. (2019) industrial, Países implementado se centra en el intercambio de residuos: el Bajos. calor residual y el $\mathrm{CO} 2$ de la empresa industrial se canalizan a los invernaderos cercanos, para apoyar el cultivo sostenible de tomates, estos residuos pasan por un sistema de tuberías para canalizar los flujos de la empresa. El objetivo de la empresa fue obtener mayor ventaja competitiva, gestionando de manera eficiente sus corrientes de residuo de calor y $\mathrm{CO} 2$, mejorando su comportamiento medioambiental y reduciendo su huella.

Morán (2019) 5 empresas, sector En Ecuador la mayoría de las empresas exportadoras son industrial, Guayaquil. agrícolas. Por lo tanto, es importante reciclar material biológico para convertirlo en un nuevo producto que contribuyan de manera positiva al sector agroindustrial y al medio ambiente.

Los indicadores a medir en las empresas son: cantidad de residuos generados por la empresa, clasificación según el tipo de residuo, reutilización del agua, reutilización de los residuos en el sistema productivo.

El modelo circular implementado genera mejoramiento de la oferta exportable a largo plazo.

Córdova $\quad 12$ empresa, sector Para el reciclaje las empresas realizan un análisis y (2019) electrónico, diagnóstico de los aparatos electrónicos, de esta manera Monterrey, determinan si el aparto puede ser reusado, mediante la México, reparación, Re-manufacturación o mantenimiento. Posterior Guadalajara. al análisis se formatea los aparatos eliminando toda información contenida en ellos, luego, se los vende al mercado de segunda mano.

Las tarjetas de circuito impreso (PCB) son exportadas para que no pierdan valor y sea recuperado en países que poseen tecnologías de procesamiento final.

Las empresas cuentan con certificaciones de proceso de reciclajeR2 y e-Stewards que indican el nivel de profesionalismo y eficacia de una empresa recicladora.

$\begin{array}{lll}\text { Zabaleta } & \text { 12 empresas, sector } & \text { Las prácticas verdes que aplican las empresas consisten en } \\ \text { pro20) } & \text { la conservación y siembra de bosques, reciclaje, } \\ \text { agroindustrial, } & \text { reutilización, reducción, utilización de aguas lluvias para los } \\ \text { artesanal, turístico, } & \text { sanitarios, energía solar. En este caso se consideran negocio } \\ \text { Colombia. } & \text { verde ya que reducen el consumo de plásticos, reciclaje, uso } \\ & \text { correcto de los recursos naturales, por consiguiente, genera } \\ & \text { bienestar del trabajador, restauración de la fauna y flora. En } \\ & \text { cuanto a la producción de servicio y bienes, buscan } \\ & \text { satisfacer las necesidades de los clientes con calidad de los }\end{array}$


productos a precio competitivos, sin causar daño al medio ambiente.

Las ventajas que obtuvieron fueron reconcomiendo frente a otras entidades por realizar prácticas medioambientales, capacitaciones por parte del ministerio del ambiente, incremento económico, generación de empleo.

Fuente: Elaboración propia a partir de los autores citados. 